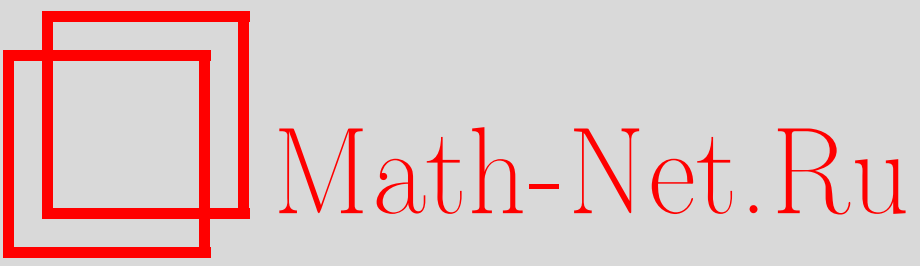

О. В. Лычагина, Нормальные формы пуассоновых структур в трехмерном пространстве, УМН, 1996, том 51, выпуск 4, 163-164

DOI: https://doi.org/10.4213/rm1005

Использование Общероссийского математического портала Math-Net.Ru подразумевает, что вы прочитали и согласны с пользовательским соглашением

http://www.mathnet.ru/rus/agreement

Параметры загрузки:

IP: 54.80 .97 .219

26 апреля 2023 г., 18:17:51 


\title{
НОРМАЛЬНЫЕ ФОРМЫ ПУАССОНОВЫХ СТРУКТУР В ТРЕХМЕРНОМ ПРОСТРАНСТВЕ
}

\author{
О.В. ЛЫЧАГИНА
}

В этой работе мы приведем формальную классификацию вырожденных пуассоновых структур в трехмерном пространстве $\mathbb{R}^{3}$. Доказательства основаны на вычислении спектральной последовательности, построенной в работе [3].

Пусть $\nabla$ - пуассонова структура в пространстве $\mathbb{R}^{3}$, обращающаяся в нуль в точке $m \in \mathbb{R}^{3}$, $\nabla_{m}=0$. Линейная часть $\nabla$ определяет структуру алгебры Ли $\mathscr{G}$ на $\mathbb{R}^{3} \simeq T_{m}^{*} \mathbb{R}^{3}$ относительно скобки $\left[d_{m} f, d_{m} g\right]=d_{m}\langle\nabla, d f \wedge d g\rangle$ для всех гладких функций $f$ и $g$ таких, что $f(m)=g(m)=0$.

Мы не рассматриваем здесь случай полупростых алгебр Ли $\mathscr{G}$ и абелевых алгебр $\mathscr{G}$. В первом случае пуассоновы структуры формально линеаризуемы [4], во втором - нахождение нормальных форм требует вычисления когомологий алгебр Ли-Склянина [3].

Пусть $\mathscr{H}=[\mathscr{G}, \mathscr{G}]-$ коммутант алгебры Ли $\mathscr{G}$, а $\mathscr{Z}(\mathscr{G})-$ ее центр.

Возможны следующие случаи [1], [2]:

(1) $\operatorname{dim} \mathscr{H}=1, \mathscr{H} \not \subset \mathscr{Z}(\mathscr{G})$.

Теорема 1. Любая вырожденная пуассонова структура $\nabla$ на $\mathbb{R}^{3}$ с линейной частью, задающей алгебру Ли типа (1), формально әквивалентна в точке т пуассоновой структуре вида $\left(x \partial_{x}+\varepsilon \cdot z^{r} \partial_{z}\right) \wedge \partial_{y}$, где $r \geqslant 2, u \varepsilon=1$ при четном $r$ u $\varepsilon= \pm 1$ при нечетном $r, a(x, y, z)$ - локальные координаты в окрестности точки $m, x(m)=y(m)=z(m)=0$.

(2) $\operatorname{dim} \mathscr{H}=1, \mathscr{H} \subset \mathscr{Z}(\mathscr{G})$.

Обозначим через $\mu \in C^{\infty}\left(\mathbb{R}^{3}\right)$ максимальный идеал, отвечающий точке $m$ :

$$
\mu=\left\{f \in C^{\infty}\left(\mathbb{R}^{3}\right) \mid f(m)=0\right\},
$$

а через $\mu_{k}, k \geqslant 1,-$ идеал функций порядка малости $k$ в точке $m$.

Пусть $\mathscr{D}=\mathscr{D}\left(\mathbb{R}^{3}\right)$ - модуль векторных полей на $\mathbb{R}^{3}$. Обозначим через $\mathscr{D}_{2}$ модуль бивекторных полей на $\mathbb{R}^{3}$ и, соответственно, через $\mu^{k} \mathscr{D}_{2}$ - подмодуль бивекторных полей, имеющих в точке $m$ порядок малости $k$.

ТЕОРема 2. Для любой пуассоновой структуры $\nabla$ на $\mathbb{R}^{3}$ с линейной частью $\nabla_{1}=$ $z \partial_{x} \wedge \partial_{y}$, задающей алгебру Ли типа (2), существует диффеоморфизм ч такой, что

$$
(\varphi)_{*}^{(2)}(\nabla)=\left(\left(z+\lambda_{1} x^{2}+\lambda_{2} y^{2}\right) \partial_{x} \wedge \partial_{y}+X_{H} \wedge \partial_{z}\right) \bmod \mu^{3} \mathscr{D}_{2},
$$

где $H=H(x, y)$ - однородньй кубический полином, а $X_{H}$ - гамильтоново векторное поле на плоскости $x, y, u(x, y, z)$ - локальнье координаты в окрестности точки $m$, $x(m)=y(m)=z(m)=0$.

(3) $\operatorname{dim} \mathscr{H}=2$.

Выберем произвольный элемент $z \in \mathscr{G}, z \notin \mathscr{H}$, и обозначим через $\lambda_{1}, \lambda_{2}$ спектр оператора $\operatorname{ad}_{z}: \mathscr{H} \rightarrow \mathscr{H}$.

ТеОрема 3. Пусть $\nabla$ - вырожденная пауссонова структура на $\mathbb{R}^{3}$ с линейной частью типа (3), а $(x, y, z)$ - локальнье координаты в окрестности точки $m, x(m)=$ $y(m)=z(m)=0$.

Тогда $\nabla$ формально әквивалентна линейной пауссоновой структуре

$$
\left(a_{11} x+a_{12} y\right) \partial_{z} \wedge \partial_{x}+\left(a_{21} x+a_{22} y\right) \partial_{z} \wedge \partial_{y}
$$

если выполнено одно из следующих условий:

1) $\lambda_{1}, \lambda_{2} \in \mathbb{R}, \lambda_{1} \cdot \lambda_{2}>0 u \frac{\lambda_{1}}{\lambda_{2}} \notin(\mathbb{N} \backslash\{1\}), \frac{\lambda_{2}}{\lambda_{1}} \notin(\mathbb{N} \backslash\{1\})$,

2) $\lambda_{1}, \lambda_{2} \in \mathbb{R}, \lambda_{1} \cdot \lambda_{2}<0 u \frac{\lambda_{1}}{\lambda_{2}} \notin \mathbb{Q}$,

3) $\operatorname{Re} \lambda_{i} \notin 0, i=1,2$. 
Используя линейные преобразования, матрицу $\left(a_{i j}\right)$ можно привести к одному из следующих видов:
a) $\left(\begin{array}{ll}1 & 0 \\ 0 & \nu\end{array}\right)$,
b) $\left(\begin{array}{ll}1 & \beta \\ 0 & 1\end{array}\right)$,
c) $\left(\begin{array}{cc}1 & \varkappa \\ -\varkappa & 1\end{array}\right)$, где $\nu, \beta, \varkappa \in \mathbb{R}$ и $\beta, \nu \neq 0$.

Теорема 4. В условиях предидущей теоремы пауссонова структура $\nabla$ формально әквивалентна одной из следующих линейных структур:

1) $\partial_{z} \wedge\left(x \partial_{x}+\nu y \partial_{y}\right)$, zде $\nu \notin \mathbb{Q}-\cup\left\{\frac{1}{n} \mid n \in \mathbb{N}, n>1\right\} \cup\{n \in \mathbb{N}, n>1\}$,

2) $\partial_{z} \wedge\left((x+\beta y) \partial_{x}+y \partial_{y}\right)$,

3) $\partial_{z} \wedge\left((x+\varkappa y) \partial_{x}+(y-\varkappa x) \partial_{y}\right)$.

\section{СПИСОК ЛИТЕРАТУРЫ}

[1] Джекобсон Н. Алгебры Ли. М.: Мир, 1964. [2] Дубровин Б. А., Новиков С. П., Фоменко А. Т. Современная геометрия. М.: Наука, 1979. [3] Lychagina O. Schouten Brackets and Lie-Sklyanin Algebras // Preprint ISBN 82-553-0962-4, № 18. University of Oslo: Institute of Math., 1995. P. 1-28. [4] Weinstein A. // J. Diff. Geom. 1983. V. 18. P. 523-557.

Принято редколлегией 18.04.1996 